\title{
PRACTICAL ASPECTS OF VASCULAR ACCESS - APPLICATION OF ECHO-DOPPLER AND ALGORHYTHM FOR MANAGEMENT
}

\author{
Veselin Petrov ${ }^{1}$, Nina Kovacheva ${ }^{1},{\text { Dilyan } \text { Petrov }^{2}, \text { Svetla Staykova }^{3} \text {, Rossen Madjov }}^{4}$ \\ ${ }^{1}$ Department of vascular surgery, ${ }^{2}$ Clinic of abdominal surgery, ${ }^{3}$ Clinic of hemodyalisis, \\ ${ }^{4}$ Department of surgery, Medical university of Varna
}

\begin{abstract}
SUMMARY
The creation and maintenance of effective and functioning vascular access together with successful management of its frequent complications cause still more close collaboration of different specialists engaged with the morbidity, hospitalisation and treatment of patients with chronic kidney diseases (CKD). The organisation of a follow-up, including adequate assessment and monitoring of clinical parameters of vascular access as well as the quality of dialysis should be unified in all dialysis centres. This proactive approach and care are expected to lower the incidence of thrombosis, infections and achieve good long term results. Imaging and functional testing methods are crucial for the duration of dialysis treatment and better quality of life for patients.

Collaboration between specialists nephrologists and other reference points (vascular surgeons, imaging specialists, general surgeons and therapists) underlies the modern algorithm for ECC treatment. Imaging and other functional, methods have a decisive significance for the duration of dialysis treatment and the good quality of life of the patients. The collaboration between nephrologists and other specialists (vascular surgeons, image specialists, general surgeons and internists) is the basis for contemporary algorithm for extracorporeal treatment.
\end{abstract}

Keywords: vascular access, dialysis, arterio-venous anastomosis, chronic kidney disease, CKD

The performance and postoperative cares of the effective vascular access in dialysis patients as well as the successful treatment of common complications, following it impose close collaboration of medical

\footnotetext{
Address for correspondence:

Veselin Petrov, MD, PhD

Clinic of Vascular Surgery

Medical University of Varna

55 Marin Drinov Str.

E-mail:vesko_petrov@abv.bg

Mobile phone: 0888358752
}

Received: August 19, 2013

Accepted: December 18, 2013 specialists (vascular surgeons, angiologists, radiologists, internists) engaged with this morbidity and hospitalisation of the patients.

Modern vascular surgery and angiology are quite concerned with prophylaxis, diagnosis and treatment of acute and chronic renal insufficiency.

Recent data show that more than 300000 people in USA need vascular access construction in order to receive adequate dialysis treatment $(11,12)$. Vascular access is still the leading cause for hospitalization and mortality among patients with chronic renal insufficiency. Patients on dyalisis need regular visits and ontime management of any complications in order to preserve the patency of vascular ac- 
cess for a possibly longest time. The vascular access for dialysis is defined as "ideal" if enough and adequate blood flow is available as well as satisfactory levels of nitrogen is found in blood. From the vascular access known up to now arteriovenous fistula is nearest to this definition.

Dialysis was once performed through long term cannulation of femoral vessels by means of Seldinger technique. In 1960 Scribner creates the external A-V shunt, and Cimino \& Brescina in 1966 constructed an anastomosis between arteria radialis and vena cephalica, which set up the beginning of a new era in vascular surgery $(6,8)$.

\section{Basic principles in planning vascular access:}

There are several basic moments for consideration of the vascular surgeon in order to guarantee the success of the operation and to assure adequate dyalisis treatment

1. When surgical treatment for construction of A-V access is indicated - patients with creatinine clearance below $25 \mathrm{ml} / \mathrm{min}$ and an increase of serum creatinine above $400 \mu \mathrm{mol} / \mathrm{l}$, or expecting the patient to be included on dyalisis up to an year. It is recommended $\mathrm{A}-\mathrm{V}$ access to be performed 6 months before the expected dyalisis treatment. During this period the kind of vascular access is to be assessed, analysed and defined. State-of-the-art vascular prostheses are available these days, which allow cannulation up to 24 hours after implantation ( AVflo ) $(9,10,12)$.

- Autogenous A-V fistulas are superior to synthetic grafts because of lower risk of rethrombosis, bleeding and infections.

- The upper extremity is a preferred place for intervention.

- Non-dominant arm and most distal anastomosis are the first choice, and in postoperative period efforts should be made to preserve v. cephalica from frequent vene punctures.

\section{Clinical assessment of arterial system}

Anamnesis for diabetes, peripheral arterial disease, claudication of upper extremity catheterisation of A-V fistula leads to damage of the arterial segment. Physical examination - palpation of pulsations, doppler examination, comparison of pressures of the two brachial arteries Allen test etc. can confirm or reject eventual involvement of the artery in complications. Any doubt for presence of stenosis should result in doppler investigation and/or arteriography. Preoperatively established arterial diameter less than $1.6 \mathrm{~mm}$ is connected with higher risk for compromising the radiocephalic fistulas. For construction of A-V fistulas in many clinics of vascular surgery arterial diameter over $2 \mathrm{~mm}$ is preferred (5).

\section{Clinical assessment of the venous system}

History of cannulation of any of the large veins, trauma or surgery on the upper limb, neck and thorax, compromised previous vascular access suggest possible venous stenosis. Stenosis of the proximal vein more than $50 \%$ is a contraindication to construct distal ipsilateral AV fistula. It is necessary to first correct the stenotic region and then to perform the anastomosis. The application of tourniquet in the brachial area and v.cephalica is a routine clinical procedure in order to identify its patency. If the vein is $2.5 \mathrm{~mm}$ or more, it is suitable for reconstruction. For clear and adequate assessment of the superficial and deep venous system Echo-Doppler ultrasound is used nowadays. These investigations provide good visualisation of the central veins $(7,10)$.

\section{Operative management}

AVF preferred in patients requiring long-term hemodialysis are as follows (12):

1. Radiocephalic with connection of radial artery and cephalic vein;

2. Brahiocephalic - connection of same vesels but in the region of the elbow;

3. Brahiobasilic - connection of radial artery and basilic vein;

4. Implantation of AV graft of synthetic material.

Special care should be taken about patients who are preparing for a kidney transplantation. Femoral dialysis catheters are not recommended in such cases $(13,14)$.

\section{Operative technique}

The decision at which level AVF should be made is taken after careful assessment of clinical and echodoppler investigation of arterieal and venous system. Usually if the cephalic vein is not damaged for any reason in the past (most often puinctures in differ- 
ent levels) a radiocephalic anastomosis of non- dominant hand should be the first choice. If these investigations show small or stenotic vein then one should try the elbow region, where the chance for a good venous runoff is bigger $(16,17)$. Anastomosis is performed using the standard vascular technique with continuous vascular suture.

It is assumed that the standard AV fistula „matures" for about 20 days, but there are cases when more time is needed. If the patient does not have a suitable vein for constructing primary AV fistula, a graft of synthetic material may be applied $(1,2,3,11)$.

\section{Postoperative monitoring}

After construction of the vascular access follow up is advised (once a month), which aims at early diagnosis of complications and timely treatment that may extend substantially the patency of the vascular access. During the check-ups, different diagnostic methods are applied such as palpation and auscultation, Doppler ultrasound and/or angiography, if necessary. The expansion of endovascular techniques increase the possibilities for correction of stenoses, which increases the vitality of vascular access for hemodialysis (16)

\section{COMPLICATIONS}

\section{Rethrombosis}

Early rethrombosis in the first 30 days is mostly due to a technical mistake. Most often the reason is insufficient inflow, stenosis of anastomosis, elongatio, tortuosity, or traction of the vein, distal venous stenosis or even thrombosis.

\section{Infection}

Infection of the vascular access site is the second reason for compromising the dialysis access. It is defined as early (before the 30th postoperative day) and late (after the $30^{\text {th }}$ day). The most common pathogen is Staphylococcus aureus. Use of temporary vascular catheters for dialysis up to 14 days after insertion can reduce infectious complications. If infection of the vascular access site is observed immediate application of a broad spectrum antibiotic is advised. Surgical management of the inflammed area together with ligation of AVF is applied $(11,12)$.

\section{True aneurysm and pseudoaneurysm}

They are found in part of the patients with autogenic AV shunts. True aneurysms and pseudoaneurysms cause serious problems in therapeutical management, especially concerning haemodialysis. They may become complicated with rupture, major bleeding or distal embolisation of thrombi. The rapid growth of the aneurysm and the appearance of skin changes are an indication for surgery, due to impending rupture. The options are excision of the aneurysm with primary reanastomosis, ligation of the aneurysm with venous bypass, partial excision and suture of the venous wall (11).

\section{CONCLUSION}

Providing an effective and long-term access for dialysis is a complex and difficult problem. Imaging and functional testing methods are predominant on the preliminary clinical screening of patients on chroniodialysis concerning the selection of operational or procedure plan. Advanced diagnostic modalities and adequate approach to the complications of dialysis access points can prolong their primary or secondary patency. Vascular access for haemodialysis is limited in time, because its main goal is to ensure the implementation of chronic dialysis to the time of renal transplantation. This depends also on several other factors:

a) diameter of inflow and outflow vessels;

b) vascular flow - the higher flow, the longer patency of the vascular access;

c) cooperation of the patient - cares and maintainance of the access;

d) general condition of the patient.

Collaboration between nephrologists and other reference points (vascular surgeons, radiologist and imaging specialists, general surgeons and therapists) underlies the modern algorithm for the extracorporeal treatment. The role of the nephrologist is to make an assessment of vascular access by means of echo-Doppler, clinical and laboratory findings. The role of the radiologist is to precise vascular access making radiological findings when needed. If there is a stenotic segment in the vascular access, the interventional radiologist can perform dilatation or recanalisation. General surgeons have limited part in 
complex treatment of vascular access, but in emergency situations - bleeding or wound infection they can give first aid and then send the patient to vascular surgery department. Therapists can help in managing concomitant diseases, usually in communication with nephrologists. After all the final aim is to achieve high volume longterm working vascular access.

\section{REFERENCES}

1. Montreuil, B. , Staykova, Sv., Denchev - Vascular and peritoneal access for dialysis - TASC criteria : STENO Varna 2006

2. Andreev, A. Vascular Imaging Techniques. In: Vascular Diseases and Applied Vascular Surgery, Sofia, Znanie, 2007, 23-26.

3. Bleyer, A.J., L. Mason, et al. A randomized, controlled trial of a new vascular catheter flush solution (minocycline-EDTA) in temporary hemodialysis access. Infect Control Hosp Epidemiol. 2005, Jun: 26 (6): 520-4.

4. Blake, P.G., R.R. Quinn, M.J. Oliver. The risks of vascular access. Kidney Int, 2012, Sep:82 (6): 623-5.

5. Bleyer, A.J., L. Mason, et al. A randomized, controlled trial of a new vascular catheter flush solution (minocycline-EDTA) in temporary hemodialysis access. Infect Control Hosp Epidemiol. 2005, Jun: 26 (6): 520-4.

6. Brescia, M.J., J.E. Cimino, K. Appell, et al. Chronic hemodialysis using venipuncture and surgically created arteriovenous fistula. J. Am. Soc. Nephrol. 1966, 10, 193, 1999

7. Cueto-Manzano, A.M., E. Rojas-Campos. Status of renal replacement therapy and peritoneal dialysis in Mexico. Perit Dial Int. 2007, Mar-Apr; 27 (2): 142-8.

8. Haire, W.D., T.G. Lynch, R.P. Lieberman, et al. Duplex scans before subclavian vein catheterization predict unsuccessful catheter placement. Arch Surg, 1992, 127-129.

9. Laird, J.R. Limitation of PTA and stentingfor the treatment of Disease of the Superficial Femoral and Popliteal Arteries. J Endovasc Ther, 2006, 13, 30-40.

10. Mickley, V. Steal syndrome-strategies to preserve vascular access and extremity. Nephrol. Dial, Transplant., 23, 2008, № 1, 19-24.
11. Montreuil,B.Vascular and Peritoneal Access Introduction, ACS Surgery Online.2002: WebMD Inc.

12. National Kidney Foundation DOQI: Clinical Practice Guidelines for Hemodialysis Vascular Access. Am J Kidney Dis 30 (suppl 3) 1997

13. Pirozzi, N., J. Scrivano, L. Pettorini, et al. Preventive hemostasis for hemodialysis vascular access surgical reinterventions. J Vasc Access. 2012, Sep, 13

14. Samama, C., et al. Venous thromboembolism prevention in surgery and obstetrics: clinical practice guidelines. European Journal of anesthesiology, 23, 2006, 95-116.

15. Scheltinga, M., F. Van Hoek, c. Bruyninckx. Surgical banding for refractory hemodialysis access-induced distal ischemia (HAIDI). J. Vasc. Access, 10, 2009, 43-49.

16. Walters, B.A.J., P. Pennell, J.P. Bosch. Hemodialysis Vascular Access and Peritoneal Dialysis Access. Quality Assurance and Continours Quality Improvement Programs for Vascular Access Care. Ronco, C., N.W. Levin (eds): Hemodialysis Vascular Access and Peritoneal Dialysis Access. Contrib Nephrol. Basel, Karger, 2004, vol. 142, pp 323-249.

17. Zamani, P., J. Kaufman, S. Kinlay. Ischemic steal syndrome following arm arteriovenous fistula for hemodialysis. Vasc. Med., 14, 2009, № 4, 371-376. 